\title{
RETRACTED ARTICLE: Variant Interleukin-1 Receptor-Associated Kinase-2 Mediates Increased NF- $\kappa B$ and p38 Activity Induced by Lipopolysaccharide
}

\author{
Li Xin, ${ }^{1}$ Wu Huiyun, ${ }^{1}$ Xiong Shanshan, ${ }^{2}$ Huang Zhisong, ${ }^{1}$ Huang Shuhai, ${ }^{1}$ Zhao Siqing, \\ Wang Hua, ${ }^{1}$ Sun Zhenhai, ${ }^{1,3}$ and $\mathrm{Xu} \mathrm{Xiegu}^{1,3}$
}

This article has been retracted by the corresponding author Li Xin due to authors' acknowledgement of academic and ethical misconduct. The authors submitted inaccurate results and did not obtain proper permission from all contributors. This is a violation of journal policy.

This article has been retracted by the corresponding author Li Xin due to authors' acknowledgement of academic and ethical misconduct. The authors submitted inaccurate results and did not obtain proper permission from all contributors. This is a violation of journal policy.

\footnotetext{
${ }^{1}$ Department of Science and Technology, Academy of Military Medical Sciences, No. 20 East Street of Fengtai District, Beijing, People's Republic of China

${ }^{2}$ Beijing Institute of Radiation Medicine, Academy of Military Medical Sciences, No. 27 North Taiping Road of Haidian District, Beijing, People's Republic of China

${ }^{3}$ To whom correspondence should be addressed at Department of Science and Technology, Academy of Military Medical Sciences, No. 20 East Street of Fengtai District, Beijing, People's Republic of China. E-mail: sunzhenh@tom.com; E-mail: xu_fortune@163.com
} 